\title{
Unit of Magnetic Permeability
}

National Cancer Institute

\section{Source}

National Cancer Institute. Unit of Magnetic Permeability. NCI Thesaurus. Code C68791.

A unit of measurement of an ability of a substance or material to acquire magnetization

in response to an applied magnetic field. 